\title{
Study on sorting method of zinc silver battery based on multi-step FCM clustering algorithm
}

\author{
Ran Li ${ }^{1 a)}$, Jie Yao ${ }^{1}$, and Yongqin Zhou $^{1}$
}

\begin{abstract}
In order to solve the problem of consistency after grouping of silver zinc battery, a multi-step FCM (MSFCM) algorithm is proposed for the separation of silver zinc battery. The method uses the clustering validity function to determine the optimal class number, two times of FCM sorting algorithm in optimal results, obtained classification results of different applications in the battery cycle life. Using the method of dynamic consistency life cycle, attenuation rate decreased significantly in the 30 cycle life test after the capacity decay rate does not exceed the idea of $10 \%$.

Keywords: silver-zinc battery, fuzzy c-mean, discharge voltage platform, consistency, rate of capacity decay
\end{abstract}

Classification: Electron devices, circuits and modules

\section{Introduction}

Zinc-silver batteries have the characteristics of high specific energy, high specific power, stable discharge voltage, good reliability and safety $[1,2]$. Therefore, it is widely used in underwater, water level equipment and aerospace [3]. Based on the demand for high-power and high-capacity batteries in these fields, the zinc-silver batteries on pack using is an effective solution [4, 5]. However, the initial performance problems between the individual cells in the battery pack are not uniform, which accelerates the degradation of battery capacity, thereby resulting in premature failure of the battery pack, and may even cause safety problems $[6,7]$. Ordinary zinc-silver battery with a service life for about 30 times will aggravate the life decay after being used in group. Therefore, it is necessary to improve the consistency of the initial performance of the participating cells by sorting before combining [8, 9].

Many researchers have focused on using the chargedischarge voltage curve as a measure of battery performance and differences, and have developed a series of methods for automatic battery sorting. Duo Zhihua et al. [10] proposed a categories algorithm based on data analysis theory for threshold criteria sorting battery, and a calculation method based on fuzzy decision-making to quickly identify battery capacity and curve consistency. Shan Yi [11], by using the hierarchical clustering method to obtain the difference between the batteries, the test showed that the

${ }^{1}$ School of Electric and Engineering, Harbin University of Science and Technology, Harbin, Heilongjiang, China

a) zeemoolr@126.com

DOI: $10.1587 /$ elex.16.20190120

Received March 2, 2019

Accepted March 8, 2019

Publicized March 22, 2019

Copyedited April 10, 2019 sorting effect of the method is better. Wen Tao et al. $[12,13]$ proposed a battery sorting method based on eigenvectors, but the standard voltage characteristic vector is more difficult to determine, which increases the difficulty of the implementation of the sorting process. Yu Zhilong et al. $[14,15,16]$ built a battery equivalent circuit model with SOC as the link, and the battery is sorted for the similarity between the charge and discharge curves based on the model simulation. Hu Xiaosong et al. $[17,18]$ conducted state estimation of lithium battery of electric vehicle's lithium battery by means of fuzzy clustering multi-model support vector machine. Guo Lei [19] and Shi Qingsheng [20] et al. carried out the sorting of lithium battery by adopting multiple algorithms such as FCM algorithm, $\mathrm{C}$-means clustering, multi-objective clustering and kernel principal component analysis [21, 22, 23, 24, 25, 26].

However, with special electrochemical properties, zinc-silver battery obviously differs from with lithium-ion battery in discharge characteristics. Thus, above methods are not applicable to zinc-silver batteries. On the basis of previous researches $[27,28]$, this paper innovatively proposed a FCM multi-stage sorting method for zinc-silver battery according to its unique characteristics of the discharge voltage platform, and then verified the method.

\section{FCM algorithm research}

\subsection{FCM algorithm}

Fuzzy C-means Algorithm (FCM) was a mature fuzzy clustering algorithm developed by Dunn [23] for the first time through Bezdek [24, 25]. The algorithm classified unlabeled data by minimizing the objective function based on a certain norm and clustering prototype. The FCM algorithm can be described as follows:

Let $X=\left\{x_{1}, x_{2}, \cdots, x_{n}\right\} \subset R^{s}$ represents a given set of cell samples, $s$ is the dimension of the sample space, $n$ is the number of samples, and $c(c>1)$ is the number of clusters that divide $X$.

The objective function of the FCM algorithm is defined as:

$$
\begin{aligned}
& J\left(U, v_{1}, \cdots, v_{c}\right)=J(U, V) \\
& =\sum_{i=1}^{c} J_{i}=\sum_{i=1}^{c} \sum_{j=1}^{n} \mu_{i j}^{m} d_{i j}^{2}, \quad m \geq 1
\end{aligned}
$$

Let $U=\mu_{i j}$ is a fuzzy partition matrix of $c \times n$ whose element $\mu_{i j}$ denotes the membership value of the $j$-th battery sample $x_{j}$ belonging to the $i$-th class. The degree 
of membership is used to indicate the extent to which a battery belongs to a certain type of battery. If its value is 1 , it means that the battery belongs to a certain type of battery. According to the normalization rule, the sum of the membership degrees of a data set is always equal to 1 , so the constraint must be met, as shown in equation (2):

$$
\begin{aligned}
& \sum_{i=1}^{c} \mu_{i j}=1, \quad 1 \leq j \leq n \\
& \sum_{j}^{n} \mu_{i j}>0, \quad 1 \leq i \leq c \\
& \mu_{i j} \geq 0, \quad 1 \leq i \leq c, \quad 1 \leq j \leq n
\end{aligned}
$$

Where: $V=\left[v_{1}, v_{2}, \ldots, v_{c}\right]$ is a matrix of $s \times c$ composed of $c$ cluster center vectors; $v_{i}$ represents the cluster center of the $i$-th battery; $d_{i j}=\left\|x_{j}-v_{i}\right\|$ represents the Euclidean distance from the sample point $x_{j}$ to the center $v_{i}, m$ is a weighted index, also known as the fuzzy factor, which determines the degree of correlation between the categories. The larger the $m$ value, the more blurred the clustering result. In the absence of special requirements, usually take $m=2$ [29].

For the independent variable $(U, V)$ constraint problem, using the Lagrangian algorithm combining equations (1) and (2), the iterative equation is obtained as shown in equations (3) and (4):

$v_{i}=\frac{\sum_{j=1}^{n} \mu_{i j}^{m} x_{j}}{\sum_{j=1}^{n} \mu_{i j}^{m}}, i=1,2, \cdots c$

$\mu_{i j}=\left[\sum_{k=1}^{c}\left(\frac{d_{i j}}{d_{k j}}\right)^{2 /(m-1)}\right]^{-1}, i=1,2, \cdots c ; j=1,2, \cdots, n$

\subsection{FCM algorithm effectiveness index}

The FCM algorithm needs to specify the number of clusters $c$ in advance, for different $c$ values the corresponding fuzzy clustering division of the algorithm is also different. The cluster validity indicator can show the quality of the obtained partitions, thus helping to determine the optimal partition. Accordingly, the effectiveness indicator can also be used to find the optimal number of clusters without prior knowing of the data structure.

In this paper, the ratio-type fuzzy clustering validity index $V_{X B}$ proposed by Wan [30] is used. The intra-class compactness is expressed as the sum of the distances of each sample to the cluster center, and the separation between classes is measured by the minimum distance between all clustering centers. The mathematical expression is expressed as equation (5):

$$
V_{X B}=\frac{J_{m}(U, V) / n}{\operatorname{Sep}(V)}=\frac{\sum_{i=1}^{c} \sum_{j=1}^{n} \mu_{i j}^{m}\left\|x_{j}-v_{i}\right\|^{2}}{n \min _{i \neq j}\left\|v_{i}-v_{j}\right\|^{2}}
$$

Where, $v_{i}$ is the $i$-th cluster center.

When the number of clusters $K$ is very large and tends to the total number of samples $n$, the $V_{X B}$ index monotonically decreases as the number of clusters increases. $V_{X B}$ is a very small indicator, that is, the number of clusters corresponding to the minimum value is the optimal number of clusters.

\subsection{Effectiveness of FCM algorithm}

In equation (4), $\mu_{i j}$ represents the degree to which the $j$-th battery sample belongs to the $i$-th class, and the equation is transformed into the equation (6).

$$
\mu_{i j}=\frac{1}{\sum_{k=1}^{c}\left(\frac{d_{i j}}{d_{k j}}\right)^{\frac{2}{m-1}}}=\frac{1}{1+\sum_{\substack{k=1 \\ k \neq i}}^{c}\left(\frac{d_{i j}}{d_{k j}}\right)^{\frac{2}{m-1}}}
$$

It is not difficult to find from equation (6) that the larger $\mu_{i j}$ is, the smaller $d_{i j}$ is, the larger $d_{k j}$ is. That is, the $j$-th battery sample $x_{j}$ is closer to the $i$-th cluster center $v_{i}$ and farther away from the other cluster centers. Therefore, measuring fuzzy membership is an important factor in evaluating the quality of clustering.

\section{Battery sorting method}

\subsection{Charge and discharge test}

In order to obtain the discharge curve of the battery sample, the cycle process as described in Table I was completed for the sample zinc-silver battery with having a capacity of $75 \mathrm{Ah}$.

Table I. Battery charge and discharge cycle process

\begin{tabular}{l|l|l}
\hline Step & Rule & End \\
\hline 1 & 9 A constant current discharge & Voltage reaches $1.0 \mathrm{~V}$ \\
\hline 2 & $\begin{array}{l}5 \text { A constant current to constant } \\
\text { voltage charging }\end{array}$ & Voltage reaches $2.05 \mathrm{~V}$ \\
\hline 3 & 9 A constant current discharge & Voltage reaches $1.0 \mathrm{~V}$ \\
\hline 4 & End, record capacity & End \\
\hline
\end{tabular}

Select a sample of zinc-silver batteries with a capacity of $75 \mathrm{Ah}$ from the same batch of a battery manufacturer as samples 1 50, and perform steps 1 4 for samples 1 50 without any treatment on samples 51 60.

\subsection{Acquisition of cluster samples}

The voltage value is extracted as the sampling point at the same time position of each battery discharge voltage platform to calculate the average value of the sampling points as the standard point, and the distance between the sampling point of the battery cell and the standard point is the absolute value of the voltage difference $\Delta u_{i j}$.

The sampling points take the voltage at the initial stable time $t 1$ voltage of the second voltage discharge platform, the $t 2$ voltage at the middle of the second voltage platform, and the $t 3$ voltage difference at the end of the plateau period which are shown in Fig. 1.

In the discharge curves of samples $1 \sim 50$, take $t_{1}, t_{2}$, and $t_{3}$ as $30 \%, 60 \%$, and $90 \%$ of the average discharge time of 50 battery samples which are shown in Table II.

The average voltage values at the three sampling points of the sampling samples $1 \sim 50$ are as shown in Table III. 


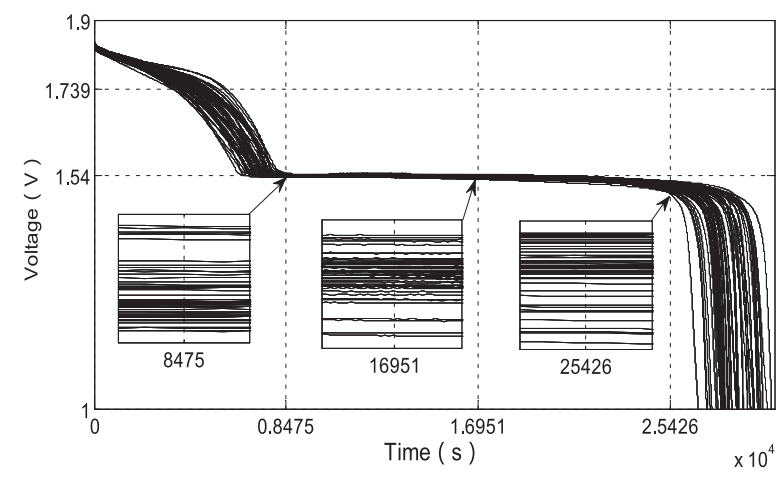

Fig. 1. Sample $1 \sim 50$ discharge curve and battery cell characteristics

Table II. Battery sampling point information

\begin{tabular}{l|l|l|l}
\hline Voltage value $/ \mathrm{V}$ & $t_{1}=8745.46 \mathrm{~s}$ & $t_{2}=16950.92 \mathrm{~s}$ & $t_{3}=25426.39 \mathrm{~s}$ \\
\hline Sample 1 & 1.537716 & 1.537494 & 1.519956 \\
\hline Sample 2 & 1.539023 & 1.538687 & 1.518575 \\
\hline Sample 3 & 1.539252 & 1.539779 & 1.524602 \\
\hline$\ldots$ & $\ldots$ & $\ldots$ & $\ldots$ \\
\hline Sample 49 & 1.538469 & 1.53715 & 1.514557 \\
\hline Sample 50 & 1.538049 & 1.537642 & 1.512856 \\
\hline
\end{tabular}

Table III. Average voltage at the sampling point

\begin{tabular}{l|l|l|l}
\hline Voltage value $/ \mathrm{V}$ & $t_{1}=8745.46 \mathrm{~s}$ & $t_{2}=16950.92 \mathrm{~s}$ & $t_{3}=25426.39 \mathrm{~s}$ \\
\hline Sample $1 \sim 50$ & $\overline{V_{1}}=1.539155$ & $\overline{V_{2}}=1.538290$ & $\overline{V_{3}}=1.516110$ \\
\hline
\end{tabular}

The voltage difference between the three sampling points is classified, and the voltage difference $\Delta u_{i j}$ is calculated as shown in equation (7).

$$
\Delta u_{i j}=v_{i j}-\overline{V_{j}}
$$

The voltage difference of the three sampling points in equation (6) is transformed into a 3-dimensional matrix, then normalized by the range transformation to obtain a 3dimensional matrix $V^{\prime}$, as shown in equation (8).

$$
\begin{aligned}
V^{\prime} & =\left(\begin{array}{c}
u_{1}^{\prime} \\
u_{2}^{\prime} \\
\vdots \\
u_{50}^{\prime}
\end{array}\right)=\left(\begin{array}{ccc}
u_{11}^{\prime} & u_{12}^{\prime} & u_{13}^{\prime} \\
u_{21}^{\prime} & u_{22}^{\prime} & u_{23}^{\prime} \\
\vdots & \vdots & \vdots \\
u_{50,1}^{\prime} & u_{50,2}^{\prime} & u_{50,3}^{\prime}
\end{array}\right) \\
& =\left(\begin{array}{ccc}
0.310327 & 0.118211 & 0.194806 \\
0.014662 & 0.057590 & 0.123391 \\
\vdots & \vdots & \vdots \\
0.235063 & 0.095783 & 0.164184
\end{array}\right)
\end{aligned}
$$

\subsection{FCM clustering algorithm results}

$V^{\prime}$ is the clustering object of the FCM algorithm. The Number of categories $c$ is taken as $2 \sim 4$, the convergence precision $\varepsilon$ is $1 \mathrm{e}-5$, and the FCM algorithm is applied to $V^{\prime}$.

\begin{tabular}{|c|c|c|c|}
\hline Category & Cluster center & $\begin{array}{l}\text { Number of } \\
\text { samples }\end{array}$ & $\begin{array}{l}\text { Categories } \\
\text { result }\end{array}$ \\
\hline 1 & $\begin{array}{l}(0.1977, \\
0.1563 \\
0.1816)\end{array}$ & 35 & $\begin{array}{l}1,2,3,4,5,7,8,10, \\
11,15,16,17, \\
18 \text { etc. }\end{array}$ \\
\hline 2 & $\begin{array}{l}(0.6274, \\
0.5330, \\
0.5148)\end{array}$ & 15 & $\begin{array}{l}6,9,12,13,14,20, \\
22,25,26,27, \\
28,29 \text { etc. }\end{array}$ \\
\hline
\end{tabular}
The categories results are shown in following Tables.
Table IV. Categories results of Number of categories $\mathrm{c}=2$

\begin{tabular}{|c|c|c|c|}
\hline Category & Cluster center & $\begin{array}{l}\text { Number of } \\
\text { samples }\end{array}$ & $\begin{array}{l}\text { Categories } \\
\text { result }\end{array}$ \\
\hline 1 & $\begin{array}{l}(0.2018, \\
0.1393 \\
0.1143)\end{array}$ & 27 & $\begin{array}{l}1,2,4,5,7,8,10 \\
11,15,17,18,19, \\
21 \text { etc. }\end{array}$ \\
\hline 2 & $\begin{array}{l}(0.6160, \\
0.2657, \\
0.5007)\end{array}$ & 11 & $\begin{array}{l}3,9,16,20,27,39, \\
40,41,42,43, \\
45\end{array}$ \\
\hline 3 & $\begin{array}{l}(0.7339, \\
0.5823 \\
0.4431)\end{array}$ & 12 & $\begin{array}{l}6,12,13,14,22, \\
25,26,28,29,31, \\
32,33\end{array}$ \\
\hline
\end{tabular}

Table V. Categories results of Number of categories $c=3$

Table VI. Categories results of Number of categories $\mathrm{c}=4$

\begin{tabular}{l|l|l|l}
\hline Category & Cluster center & $\begin{array}{l}\text { Number of } \\
\text { samples }\end{array}$ & $\begin{array}{l}\text { Categories } \\
\text { result }\end{array}$ \\
\hline \multirow{3}{*}{1} & $\begin{array}{l}(0.2030, \\
0.1319, \\
0.0930)\end{array}$ & 24 & $\begin{array}{l}1,2,4,5,7,8,10, \\
11,15,17,18,19, \\
23 \text { etc. }\end{array}$ \\
\hline \multirow{3}{*}{2} & $(0.1689$, & & $3,16,21,27,30$, \\
& 0.1930, & 12 & $37,39,40,41,42$, \\
3 & $0.3722)$ & & 43,45 \\
\hline \multirow{3}{*}{4} & $(0.4639$, & & $6,9,13,14,20,22$ \\
& 0.5033, & 6 & $12,25,26,28,29$, \\
& $0.7270)$ & & $31,32,33$ etc. \\
\hline
\end{tabular}

The calculated validity function value $V_{X B}$ is expressed in Table VII.

When the number of categories $\mathrm{c}$ is $2 \sim 4$, the categories effect of samples 1 50 is shown in Fig. 2 4.

When the number of categories $c$ is $2 \sim 4$, the validity function $V_{X B}$ takes the smallest value when $c=3$, so the best number of categories is $c=3$. The first type of cluster centers at $c=3$ are numerically smaller than the second and third categories, indicating that the voltage difference between the 27 samples of the first type is the smallest, and the consistency of the pack is the best. Therefore, it can be as the optimal results.

Since the number of categories $c$ is too large, the application of battery sorting into packs is of little significance and will not be discussed in this paper.

\subsection{MSFCM algorithm}

The capacity type battery and the power type battery having different discharge characteristics can be separately 
Table VII. Value of the validity function

\begin{tabular}{l|l|l|l}
\hline $\begin{array}{l}\text { Number of } \\
\text { categories c }\end{array}$ & 2 & 3 & 4 \\
\hline$V_{X B}$ & 1.94650 & 0.27729 & 0.59876 \\
\hline
\end{tabular}

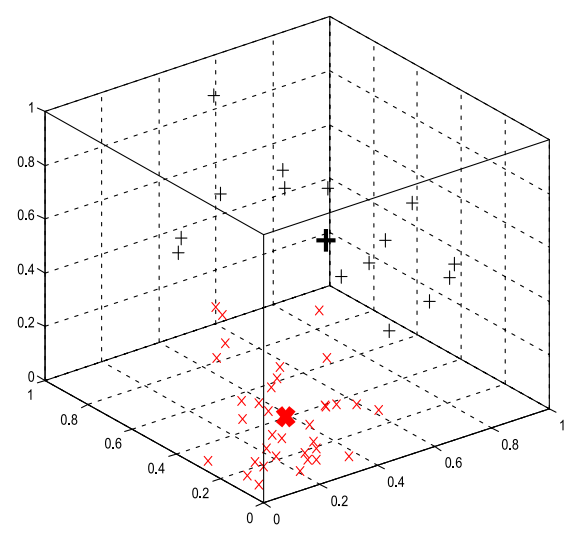

Fig. 2. Number of categories $c=2$

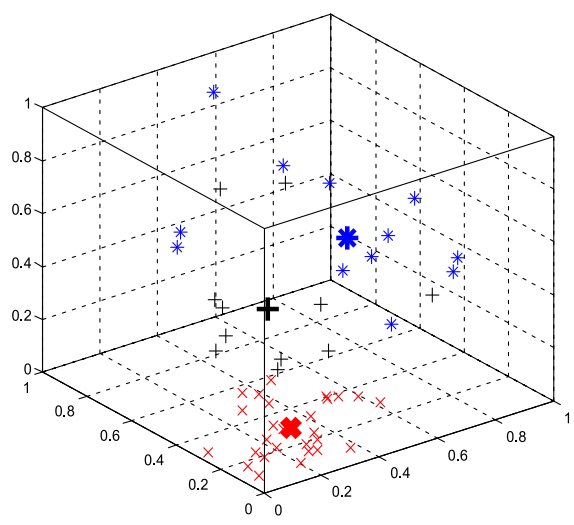

Fig. 3. Number of categories $c=3$

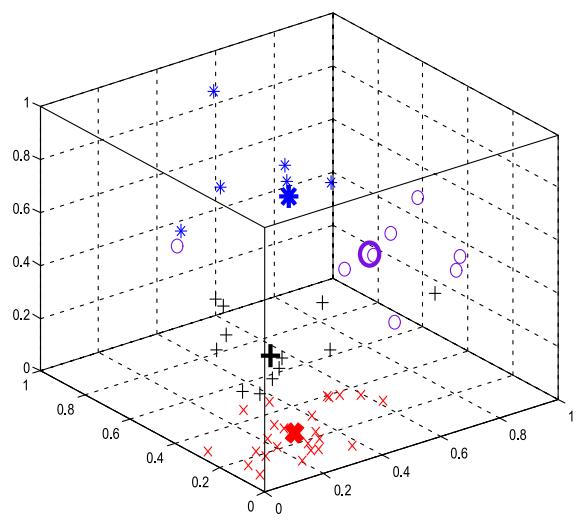

Fig. 4. Number of categories $c=4$

sorted according to the actual needs of the project and the characteristics of the first voltage platform. The power type zinc-silver battery can maintain a relatively high voltage during the first discharge stage, but at the end of the discharge phase, the earliest linear decline trend occurs; the second discharge platform of the capacity type battery maintains a long time, and finally end discharges.
The discharge time of $V_{1}=1.739 \mathrm{~V}$ and the discharge time of $V_{2}=1.10 \mathrm{~V}$ will be taken, when the optimal category result (the first type of 27 samples when the number of category $c=3$ expressed in Table V) is taken. As a quadratic clustering object, the categories of two types of batteries can be obtained by FCM clustering algorithm. The data samples are selected as shown in Fig. 5.

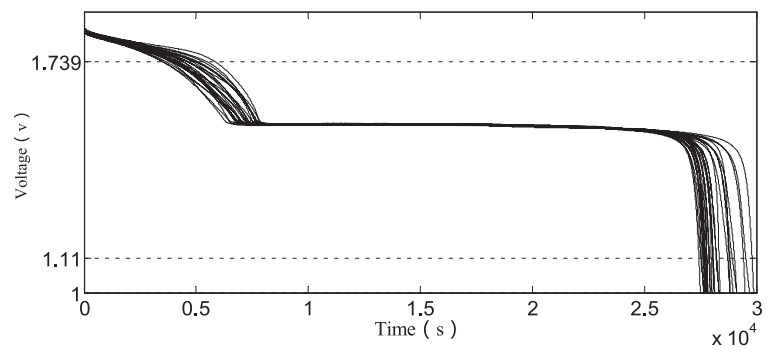

Fig. 5. Data sample selection for twice clustering

Get the data samples as shown in Table VIII.

Table VIII. Battery sampling point information

\begin{tabular}{l|l|l}
\hline Time $/ \mathrm{s}$ & $V_{1}=1.739 \mathrm{~V}$ & $V_{2}=1.110 \mathrm{~V}$ \\
\hline Sample 1 & 4638 & 29526 \\
\hline Sample 2 & 5514 & 28920 \\
\hline Sample 3 & 5115 & 28212 \\
\hline$\ldots$ & $\ldots$ & $\ldots$ \\
\hline Sample 26 & 4068 & 27630 \\
\hline Sample 27 & 3978 & 27429 \\
\hline
\end{tabular}

The standardized formula is as shown in equations (9) to (10), and the normalized data samples are as shown in equation (11).

$$
\begin{aligned}
& t_{i j}^{\prime}=\frac{t_{i j}-\min \left\{t_{i j} \mid 1 \leq i \leq 27\right\}}{\max \left\{t_{i j} \mid 1 \leq i \leq 27-\min \left\{t_{i j} \mid 1 \leq i \leq 27\right\}\right.}, \quad j=1 \\
& t_{i j}^{\prime}=\frac{\max \left\{t_{i j} \mid 1 \leq i \leq 27\right\}-t_{i j}}{\max \left\{t_{i j} \mid 1 \leq i \leq 27-\min \left\{t_{i j} \mid 1 \leq i \leq 27\right\}\right.}, \quad j=2
\end{aligned}
$$

$$
\begin{aligned}
T^{\prime} & =\left(\begin{array}{cc}
t_{11}^{\prime} & t_{12}^{\prime} \\
t_{21}^{\prime} & t_{22}^{\prime} \\
\vdots & \vdots \\
t_{27,1}^{\prime} & t_{27,2}^{\prime}
\end{array}\right) \\
& =\left(\begin{array}{cc}
0.471117 & 0.117424 \\
0.845956 & 0.372475 \\
\vdots & \vdots \\
0.188703 & 1
\end{array}\right)
\end{aligned}
$$

In equation (11), $T^{\prime}$ is the clustering object of the MSFCM algorithm. Through MATLAB programming, the categories effect is shown in Fig. 6. The sample point closer to $(1,1)$ is the power type battery, and the sample point closer to $(0,0)$ is the capacity type battery. 


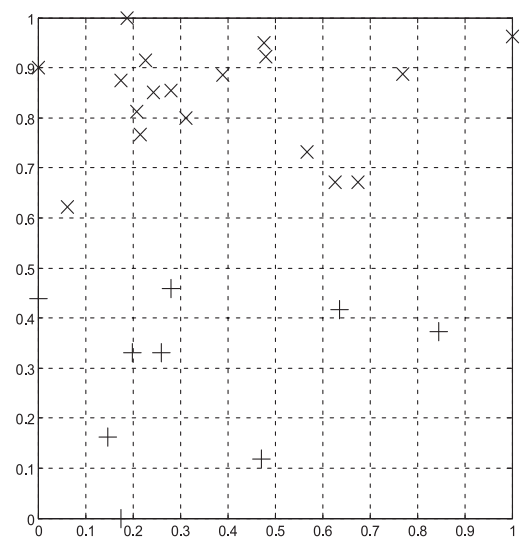

Fig. 6. Cluster categories effect

Table IX. Secondary cluster categories results

\begin{tabular}{l|l|l|l}
\hline Category & Cluster center & Number of samples & Categories result \\
\hline 1 & $(0.308917$, & 9 & $1,2,10,15,19,23,30,37$ \\
\hline 2 & $0.329486)$ & & $\begin{array}{l}4,5,7,8,11,17,35,36,38,44 \\
\text { etc. }\end{array}$ \\
\hline
\end{tabular}

The categories results are shown in Table IX. In summary, based on the first clustering, combined with the first discharge voltage platform, the second cluster is performed at different times of the same voltage, and two packs batteries with good consistency (power type and capacity type) are selected and can be applied to different application fields.

\section{Algorithmic verification}

The design test paralleled the batteries into packs to verify the effectiveness of the sorting method. Random extraction of 3 parallels in the first category, 3 parallels from unsorted samples 51 55. And the same extraction discipline for the second category.

During the test, when the number of cycles of charge and discharge of the battery pack reached 30, the test was terminated, and the capacity degradation ratio of each battery pack at the end of the test was obtained, as shown in Table X.

The test results of battery packs $1 \sim 4$ are summarized and analyzed, as shown in Fig. 7.

The cycle life of the battery packs 1 and 3 obtained by the sorting method is higher than that of the battery pack 2 and 4 .

Tests show that the categories result of the secondary separation method used in this study is effectively controlled by the cycle life degradation rate, and the capacity retention capacity of the battery pack without using any sorting method is significantly improved. When focusing on certain specific applications, it is possible to carry out three-level or multi-stage sorting based on the categories results of the second-level sorting, and combine the battery concerned performance parameters and the actual engineering requirements, and the pack is highly safe, high consistency, high reliability zinc-silver power battery pack.
Table X. Cycle life test results

\begin{tabular}{l|l|l|l|l}
\hline Test pack & 1 & 2 & 3 & 4 \\
\hline Capacity degradation (\%) & 9.55 & 18.31 & 15.80 & 36.90 \\
\hline
\end{tabular}
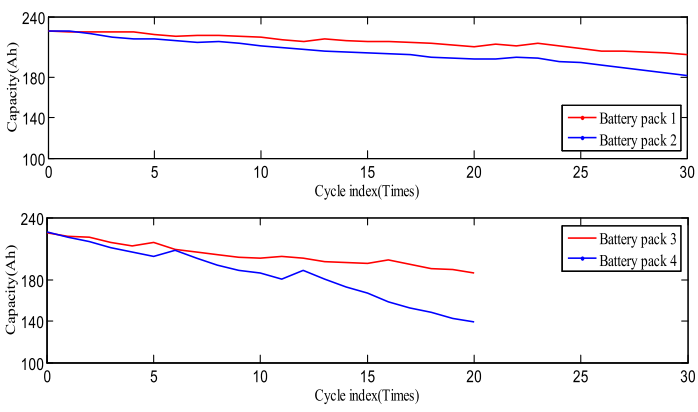

Fig. 7. Battery pack 1 4 capacity degradation curve

\section{Conclusion}

For the inconsistency of zinc-silver battery in pack use, based on the relationship between battery capacity degradation and discharge voltage curve, a MSFCM sorting method based on discharge voltage platform was proposed. This method can be used to comprehensively evaluate the quality of battery classification, select the appropriate number of classifications, realize the optimized sorting of batteries, and prolong the service life of battery packs. The test results showed that the cycle life degradation rate of the battery pack obtained by the sorting method is reduced, and the dynamic consistency is good. The optimal categories result remains at $90.45 \%$ after 30 cycles of life test. MSFCM algorithm with high precision in big data sample conditions will be applied to the sorting of small-sized samples and the study of different working conditions in the future.

\section{Acknowledgments}

This work is supported by the National Key Research Program of China (2016YFC0300104) and the Equipment Pre-research Project of China (41421040301).

\section{References}

[1] X. Jin: "Application and development of zinc silver battery," Power Technol. 12 (2011) 1613.

[2] M. Sabzi and S. M. Dezfuli: "A study on the effect of compositing silver oxide nanoparticles by carbon on the electrochemical behavior and electronic properties of zinc-silver oxide batteries," Int. J. Appl. Ceram. Technol. 15 (2018) 1446 (DOI: 10.1111/ijac. 13047).

[3] R. Ubelhor, et al.: "Enhanced thermal property measurement of a silver zinc battery cell using isothermal calorimetry," Thermochim. Acta 606 (2015) 77 (DOI: 10.1016/j.tca.2015.03.013).

[4] L. J. Giltner: "Silver-zinc batteries in marine applications," Challenges of Our Changing Global Environment. Conference Proc. 2 (1995) 803 (DOI: 10.1109/OCEANS.1995.527310).

[5] J. M. Lee, et al:: "Fabricating a continuous fiber silver-zinc battery with micro-sized diameter," ChemElectro 5 (2018) 3361 (DOI: 10.1002/celc.201801005).

[6] M. Senthilkumar, et al.: "Effect of temperature and charge stand on 
electrochemical performance of silver oxide-zinc cell," J. Energy Storage 6 (2016) 50 (DOI: 10.1016/j.est.2016.02.008).

[7] A. Yin, et al.: "Driving range estimation for battery electric vehicles based on driving cycle identification," Qiche Gongcheng/ Automot. Eng. 36 (2014) 1310.

[8] M. Ghofrani, et al:: "Optimal charging/discharging of grid-enabled electric vehicles for predictability enhancement of PV generation," Electr. Power Syst. Res. 117 (2014) 134 (DOI: 10.1016/j.epsr 2014.08.007).

[9] Y.-H. Chen, et al.: "Study on sorting technology for lithium-ion power battery of electric vehicle," J. Hunan Univ. Nat. Sci. 43 (2016) 23.

[10] Z. Duo, et al: "A fast battery classification algorithm for waveform recognition,” J. Harbin Univ. Sci. Technol. 6 (2001) 52 (DOI: 10. 15938/j.jhust.2001.04.015)

[11] S. Yi: Research on Consistency of Lithium Ion Battery (Shanghai Institute of Microsystems and Information Technology, Chinese Academy of Sciences, Shang Hai, 2008)

[12] T. Wen, et al:: China Patent 201110367124X (2012).

[13] Q. Wang, et al.: "Spectral partitioning and fuzzy C-means based clustering algorithm for big data wireless sensor networks," EURASIP J. Wirel. Commun. Netw. 2018 (2018) 54 (DOI: 10. 1186/s13638-018-1067-8).

[14] Z. Yu, et al:: "Research for classification method of battery based on state of health,” Int. J. Sec. Appl. 10 (2016) 47 (DOI: 10.14257/ ijsia.2016.10.6.06)

[15] W. Yan, et al.: "A novel combined data mining algorithm for stateof-charge estimation in electric vehicle," Int. J. Adv. Comput. Technol. 4 (2012) 662 (DOI: 10.4156/ijact.vol4.issue19.79).

[16] D. Gharavian, et al:: "ZEBRA battery SOC estimation using PSOoptimized hybrid neural model considering aging effect," IEICE Electron. Express 9 (2012) 1115 (DOI: 10.1587/elex.9.1115).

[17] X. Hu, et al.: "Fuzzy clustering based multi-model support vector regression state of charge estimator for lithium-ion battery of electric vehicle," 2009 International Conference on Intelligent Human-Machine Systems and Cybernetics (2009) 392 (DOI: 10.1109/IHMSC.2009.106).

[18] X. Liu, et al:: "Optimal sizing for wind/PV/battery system using fuzzy c-means clustering with self-adapted cluster number," Int. J. Rotating Mach. 2017 (2017) 5142825 (DOI: 10.1155/2017/ 5142825).

[19] L. Guo and G. W. Liu: "Research of lithium-ion battery sorting method based on fuzzy C-means algorithm," Adv. Mater. Res. 354 (2012) 983 (DOI: 10.4028/www.scientific.net/AMR.354-355.983).

[20] Q. S. Shi and X. Z. Zheng: "Electric vehicle charging stations optimal location based on fuzzy C-means clustering," Appl. Mech. Mater. 556-562 (2014) 3972 (DOI: 10.4028/www.scientific.net/ AMM.556-562.3972).

[21] C.-C. Kung and S.-C. Chang: "The estimation of the state of charge for lithium-ion battery by fuzzy c-regression model (FCRM) clustering algorithm," IEEE International Conference on Systems, Man and Cybernetics (2011) 1568 (DOI: 10.1109/ICSMC.2011. 6083894)

[22] Z. Shan, et al.: "Study of multi-prototype fuzzy C-means clustering algorithm using correspondence analysis," J. Huazhong Univ. Sci. Technol. (Nat. Sci. Ed.) 40 (2012) 107.

[23] X. Hu, et al.: "Advanced machine learning approach for lithium-ion battery state estimation in electric vehicles," IEEE Trans. Transport. Electrific. 2 (2016) 140 (DOI: 10.1109/TTE.2015.2512237).

[24] Z. Su, et al.: "A novel vehicle fuel cell fault diagnosis method based on FCM," Mechatronics 22 (2016) 3 (DOI: 10.16413/j.cnki.issn. 1007-080x).

[25] M. A. Khalilia, et al: "Improvements to the relational fuzzy cmeans clustering algorithm," Pattern Recognit. 47 (2014) 3920 (DOI: 10.1016/j.patcog.2014.06.021).

[26] T. Lee, et al.: "K-maximin clustering: A maximin correlation to partition-based clustering," IEICE Electron. Express 6 (2009) 1205 (DOI: 10.1587/elex.6.1205).

[27] R. Li, et al: "Lithium iron phosphate battery sorting research based on fuzzy clustering analysis," ICIC Express Lett. Part B Appl. 4 (2013) 943.
[28] Z. Rui, et al.: "A battery sorting scheme based on fuzzy C-mean clustering, taking advantage of the flatness of discharge voltage curve," Automot. Eng. 39 (2017) 864 (DOI: 10.19562/j.chinasae. qcgc.2017.08.003).

[29] K. Ping, et al:: "Research on fast fuzzy clustering algorithm based on FCM," J. Electron. Meas. Instrum. 2 (2007) 15 (DOI: 10.13382/ j.jemi.2007.02.020).

[30] S. Wan, et al.: "Fault diagnosis for high voltage circuit breaker based on timing parameters and FCM," IEICE Electron. Express 15 (2018) 20180227 (DOI: 10.1587/elex.15.20180227). 